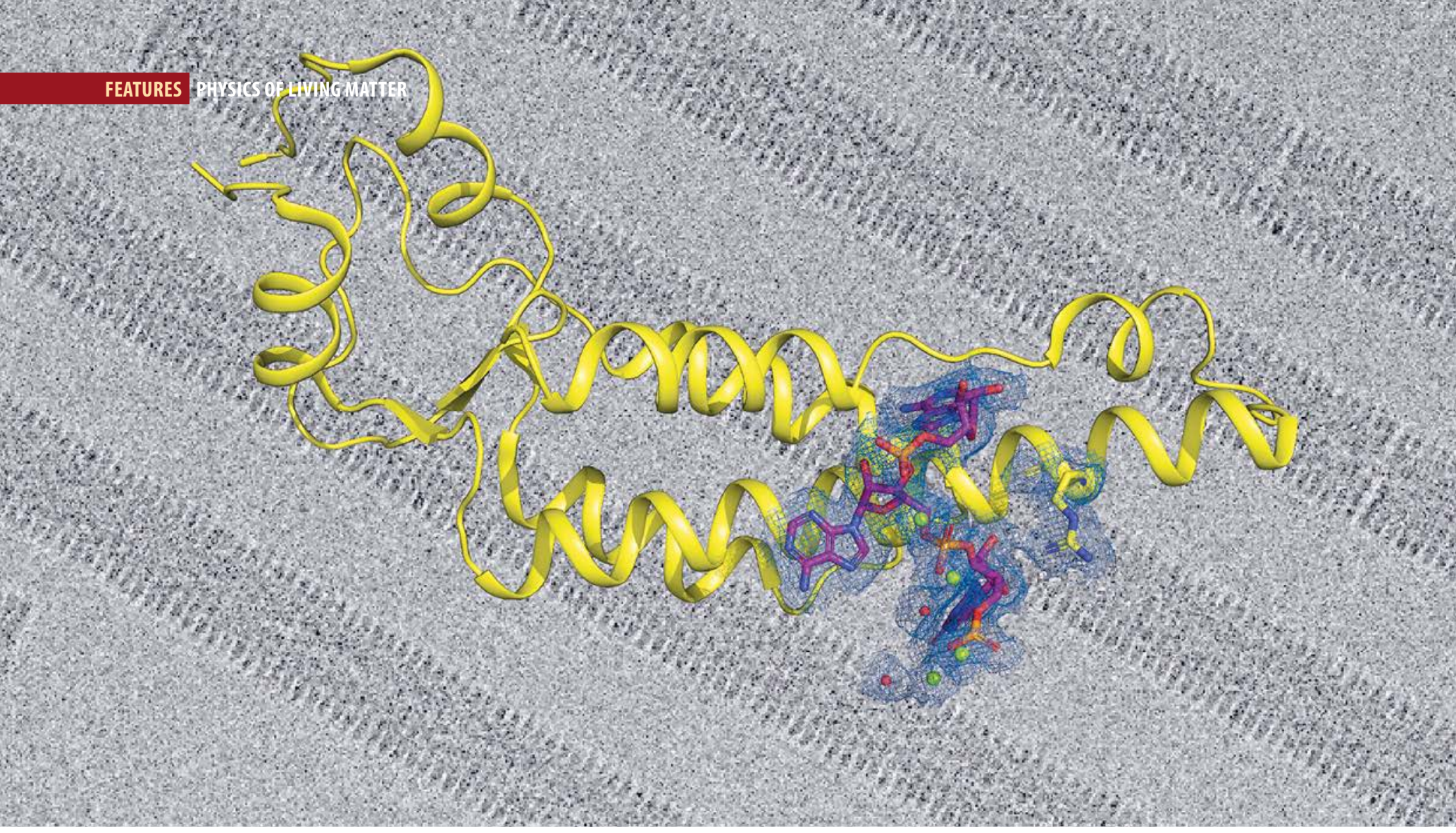

\title{
SEEING WITH ELECTRONS
}

I Arjen J. Jakobi - https://doi.org/10.1051/epn/2020502

- Department of Bionanoscience, Kavli Institute of Nanoscience, Delft University of Technology, The Netherlands

\begin{abstract}
Transformative technological advances have propelled cryogenic electron microscopy (cryo-EM) to take center stage in elucidating the intricacies of the nanoscale molecular machinery of viruses, bacteria and eukaryotic cells. Continued developments hold exciting promise for structural biophysicists to move closer to their dream of visualising atomic resolution snapshots of individual molecules at work in their native cellular environment.
\end{abstract}

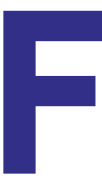

rom its invention in the 1930s by Ernst Ruska and colleagues, the electron microscope has provided biologists with a tool to visualise biological structures at resolutions beyond the limits set by the optical microscope. It has since become an indispensable instrument for determining the structure of biological materials of all kinds, and has greatly contributed to the description of cellular ultrastructure and the molecular machinery underlying cellular function. The central appeal of "seeing with electrons" is that once we can visualise the cellular machinery at a level that allows discerning the structures of its molecular components, we can describe it with the laws of chemistry and physics to answer how and why its molecular components work the way they do.

\section{Freezing water without forming ice}

Despite the early successes, the application of electron microscopy in biology has long been limited by the nature of biological samples. The key issue is that electrons scatter strongly from air, which requires conducting the imaging experiment in a high vacuum. Biological materials, which naturally exist in a watery environment, must therefore be dried or otherwise fixated before they can be imaged. Modern three-dimensional (3D) electron microscopy as it is used today for visualising the architecture of biomolecular assemblies began with the introduction of a unique specimen preparation method by Jacques Dubochet and coworkers in the 1980s. Their method formed the basis for preserving biological molecules in solution while observing them in the electron 
microscope [1]. This is achieved by cooling a thin liquid film containing the molecules so rapidly that the layer passes through its glass-transition temperature and forms an amorphous solid phase that is thought to largely preserve the native structure of water. This amorphous ice layer is maintained at liquid nitrogen temperatures during the imaging experiment, from which the technique lends its name: cryogenic electron microscopy, or cryo-EM. The molecules suspended in the ice layer are directly visualised by passing highenergy electrons through the sample and recording twodimensional (2D) phase-contrast projection images on a suitable detector. The images provide rich information, but pose challenges to interpretation because they represent the superposition of the $3 \mathrm{D}$ structure onto a single plane.

The central goal of cryo-EM is therefore to recover the 3D information by reconstructing a high-resolution estimate of the 3D electrostatic potential of the suspended biomolecules from a set of such images
(Figure 1). Its practical realisation is complicated by two compounding challenges: (1) The three-dimensional location and orientation of the molecules within the ice layer are random and unknown, and (2) the maximum tolerable dose for illumination is severely limited to prevent damage of the radiation-sensitive biological material by the high-energy electron beam. As a result, cryo-EM produces images with extremely poor contrast in which the power of the signal may be more than ten times smaller than the power of the noise. These conditions transform tasks such the detection and alignment of particles, and the assignment of relative orientations into a formidable challenge. As a result, cryo-EM has long been restricted to the study of large and highly symmetric specimens that, based on their size and symmetry facilitated alignment and reconstruction.

\section{From blobology to atomic resolution}

Due the limited resolution caused by the poor image contrast, cryo-EM was long referred to as blobology by less

V FIG. 1: Structure determination by single-particle cryo-EM. A series of images corresponding to 2D projections of individual molecules are localised and extracted from a set of electron micrographs (1). The Fourier transform of each of these 2D projections (2) corresponds to a central section of the 3D transform originating from the 3D structure. Once all 2D transforms have been correctly placed by assigning their relative orientations (3), the original 3D structure can be reconstructed by the inverse Fourier transform leading to an initial 3D model (4). Simulated 2D projections can be generated from this model (5) and used to improve assignment of relative orientations of the original data in an iterative process (2-6) until the process converges (7). If resolution is sufficiently high the resulting estimate of the molecule's 3D electrostatic potential can be used to build an atomic model (8).
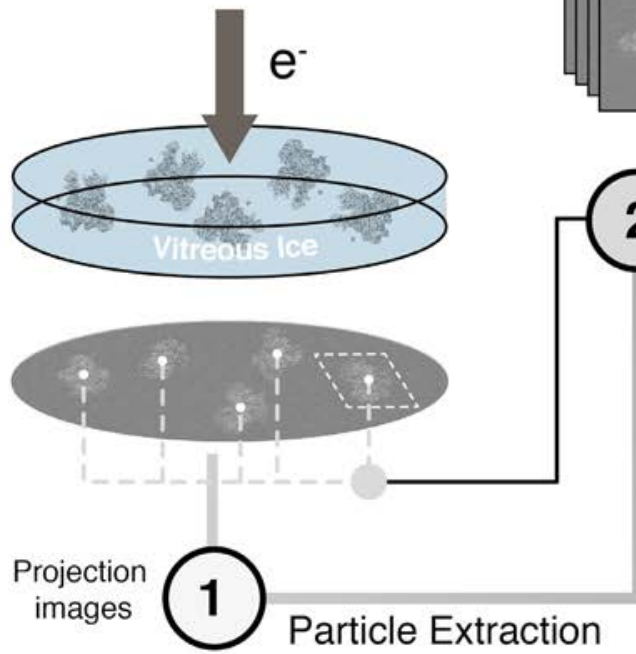

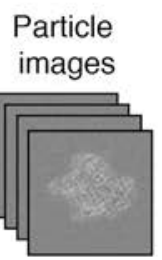

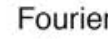

slices

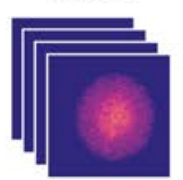

3D transform

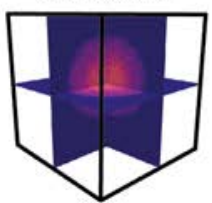

(initial)

3D model 
The future looks bright for electron

microscopy to help realise the biophysicists'

dream to watch, learn and comprehend

the inner workings of the molecular

machinery in cells and at atomic detail.on

benevolent structural biologists. Unyieldingly, electron microscopists continued to push the development of their technique encouraged by the prediction that, theoretically, it should be able to reach atomic resolution once a number of technical challenges can be overcome [2]. True to this prediction, recent advances in instrumentation and software have led to spectacular improvements of the potential of cryo-EM to produce $3 \mathrm{D}$ structures of biomolecular complexes at atomic detail, a realm long reserved to other structural biology techniques such as X-ray crystallography and nuclear magnetic resonance (NMR). The advancement that propelled cryo-EM from "blobology" to a high-resolution technique was largely driven by the development of direct electron detection (DED) cameras based on complementary metal oxide semiconductor (CMOS) technology [3]. These cameras provided two key advantages: (1) they can convert charges of electrons striking the detector directly into signal without first converting the electron charge into photons in a phosphor scintillator as is the case for CCD cameras.
This allows localising the impinging electrons with much higher precision and together with lower readout noise leads to dramatic improvements in image contrast. (2) Instead of recording a single image per exposure, the large frame rate of the DEDs allows the recording of "movies" which provides the possibility to correct for specimen movement due to drift or charging that lead to blurring of the image and hence loss of resolution.

The quality of a reconstruction also depends on the ability to discern different structural and compositional states of the target molecule(s) captured in the images, and the accuracy of image alignment (i.e. the assignment of orientations) of all particles used to reconstruct the $3 \mathrm{D}$ density map. The poor signal-to-noise ratio of cryo-EM images poses challenges to deterministic methods for particle alignment because individual particles provide insufficient contrast to allow the assignment of a unique orientation. This realisation has led to the development of probabilistic approaches that can account for such uncertainty by providing Bayesian, or maximum likelihood, estimates of orientation parameters, and proved more robust in classifying subsets of particle images with similar conformations out of a dataset containing many different structural states of the target molecule [3].

These advances have opened up the possibility to approach many challenging biological questions that were considered too difficult to be studied structurally only a few years ago. Foremost, the improved image contrast allows solving biomolecular structures of much lower molecular weight and symmetry then previously deemed possible. This has transitioned cryo-EM away from being a method applicable to only

VFIG. 2: The cryo-EM "revolution" in numbers. (a) The cumulative number of 3D cryo-EM structures displayed as released entries at the Electron Microscopy Data Bank. (b) Maximum map resolution of cryo-EM structures from single-particle averaging (SPA, left) and sub-tomogram averaging (STA, right). The dashed grey line indicates the year in which DED cameras entered the field. (c) Distribution of molecular weight (top) and average map resolution (bottom) of all entries in the Electron Microscopy Data Bank.

a

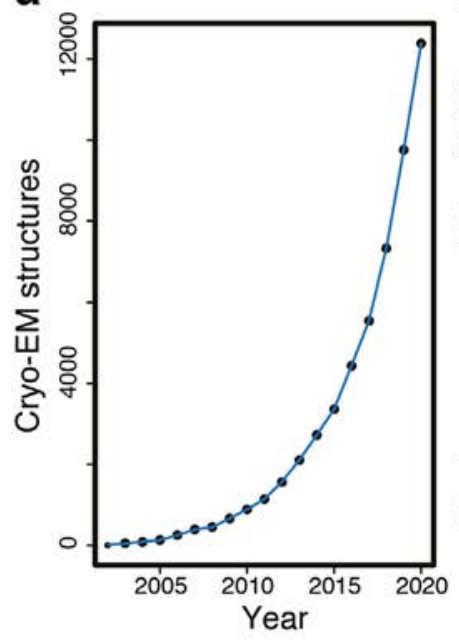

b

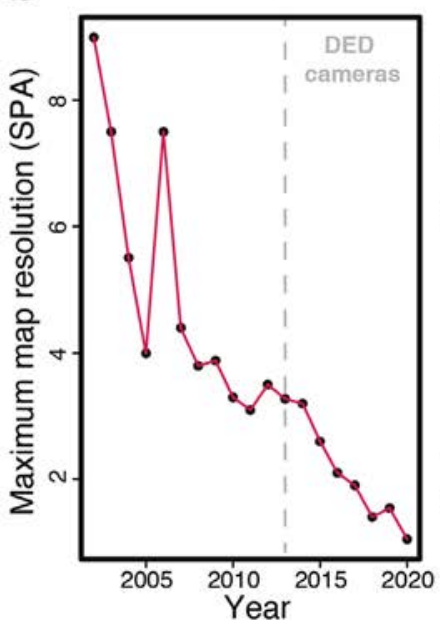

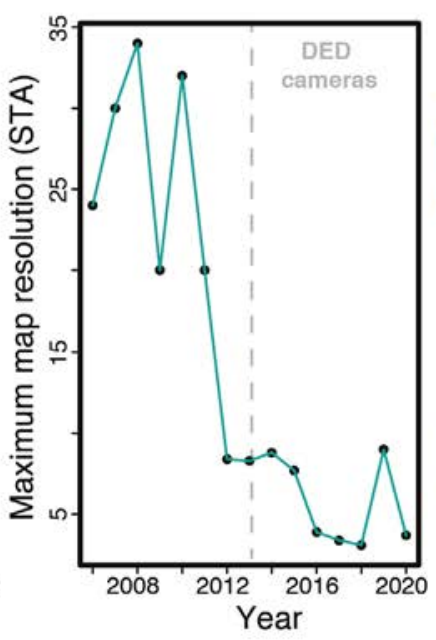

c

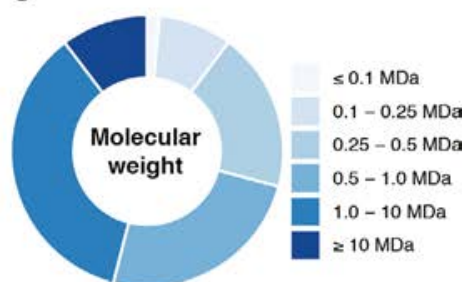

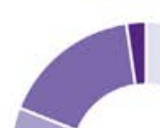

$5 \AA$

$5-10 \AA$

$10-20 \AA$

$20-60 \AA$

$\geq 60 \AA$ 
a small subset of specimens to becoming the method of choice for structure determination of a large spectrum of proteins and macromolecular assemblies central to biology. Overall, it has led to an exponential increase in the number of biomolecular structures solved by cryo-EM. It has also opened up a race of solving structures to the highest possible resolution, the record currently held at $\sim 1.2 \AA$ (Figure 2). This ambition is well-founded: such structures provide insight that can inform important mechanistic questions of enzymatic reactions, or allow revealing structural details of drug binding sites essential for the development of new medicines. Once such resolutions can be achieved reliably, cryo-EM may take a central role in pharmaceutical drug discovery.

\section{Beyond static structures}

The goal of maximising resolution often comes with the effort to limiting the compositional and structural heterogeneity of the target molecule through biochemically stabilising or physically arresting a particular structural state. With respect to promoting our fundamental mechanistic understanding of biology this effort is unfortunate, as structural variability is often important for biological function and the ability to simultaneously image the full spectrum of functional states is a unique advantage that cryo-EM over other structural techniques. Fortunately, the success of probabilistic methods to efficiently sort structural heterogeneity has led electron microscopists to appreciate this rich information hidden in their data, and it is now common that many uniquely discernible structures are reported from a single cryo-EM experiment. Improvements in sample preparation methods can be expected to facilitate capturing these structural states and movements step-by-step as they interconvert, providing the opportunity to assemble still images into molecular movies that describe the full functional cycle of biomolecular machines at atomic detail.

\section{Towards structural biology in situ}

Despite these exciting prospects, single-particle cryo-EM still requires at least partial purification of target molecules away from their native environment inside cells. Another exciting development therefore is the rapid progression of a specialised discipline of cryo-EM, cryogenic electron tomography and the associated method of sub-tomogram averaging. In electron tomography, images containing different views of the same specimen are generated by tilting the sample along an axis perpendicular to the electron beam to create $3 \mathrm{D}$ reconstructions of target molecules directly inside cells [5]. Yet, this method suffers from the drawback that an equivalent electron dose must now be distributed over many images of the tilt series, leading to images with even poorer contrast. In a process called sub-tomogram averaging, different copies of identical molecules retrieved from many tomograms can be aligned in $3 \mathrm{D}$ and averaged together to increase resolution. In several cases, this method has yielded structures at resolutions comparable to those achieved in average single-particle experiments, with the key advantage of visualising molecular interactions and modifications as they occur in their native cellular context [6]. To routinely yield high-resolution snapshots of target molecules in this way will require additional technological advances that further improve image contrast and accuracy of reconstruction algorithms, that increase throughput by automation and that facilitate a robust integration of electron microscopy with methods such as single-molecule fluorescence and super-resolution microscopy. Once established, the future looks bright for electron microscopy to help realise the biophysicists' dream to watch, learn and comprehend the inner workings of the molecular machinery in cells and at atomic detail.

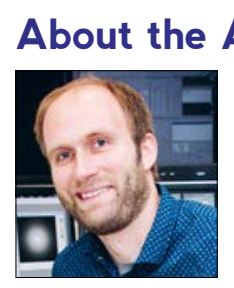

Author

Arjen Jakobi is an assistant professor at the Kavli Institute of Nanoscience at Delft University of Technology. He and his team develop methods to help cryo-EM realise its full potential.

\section{References}

[1] M. Adrian, J. Dubochet, J. Lepault and A.W. McDowall, Nature 308, 32 (1984)

[2] R. Henderson, Q. Rev. Biophys. 28, 171 (1995)

[3] G. McMullan, A. Clark, R. Turchetta and A. Faruqi, Ultramicroscopy 109, 1411 (2009)

[4] S. H. W. Scheres, J Struct Biol. 180, 519 (2012)

[5] O. Medalia, I. Weber, A. S. Frangakis, D. Nicastro, G. Gerisch and W. Baumeister, Science 298, 1209 (2002)

[6] F. J. O'Reilly, X. Liang, A. Graziade, L. Sinn, S. Lenz, D. Tegunov, C. Blötz, N. Singh, W. J. H. Hagen, P. Cramer, J. Stülke, J. Mahamid and J. Rappsilber, Science 369, 554 (2020)

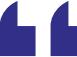
The advancement that propelled cryo-EM from "blobology" to a high-resolution technique was largely driven by the development of direct electron detection (DED) cameras. $\eta 7$ 